\section{Physician resistance to obesity pharmacotherapy}

To the Editor: Obesity, excessive body fat that impairs health, is defined by a body mass index (BMI) greater than $30 \mathrm{~kg} / \mathrm{m}^{2} .{ }^{1}$ Although the prevalence of obesity has dramatically increased in the United States, treatment of obese Americans remains suboptimal. Stigma, costs, and physician lack of confidence in medical management of obesity may all contribute to this state of affairs. ${ }^{1}$

In a survey of 13,158 obese patients, ${ }^{2}$ only $40.4 \%$ received weight-loss counseling. In another study of 45 physicians, ${ }^{3}$ most reported calculating patient BMI, but only $13.5 \%$ consistently discussed the results with their patients. ${ }^{3}$ Weight bias, including concerns about insulting patients, may contribute to physicians' reluctance to discuss obesity as a legitimate health concern. ${ }^{1}$

Further, providers who broach the subject often recommend lifestyle interventions. One survey showed that physicians were more comfortable counseling about lifestyle modification and least comfortable discussing pharmacotherapy. ${ }^{3}$ Physi-cal activities, while essential, may not be sufficient to maintain weight loss over time. This is due to compensatory physiology that promotes weight maintenance.

In theory, patients with a BMI greater than $30 \mathrm{~kg} / \mathrm{m}^{2}$ or a BMI greater than $27 \mathrm{~kg} / \mathrm{m}^{2}$ with obesity-related complications who do not respond to a healthy low-calorie regimen and physical activity are eligible for medications. In practice, only $2 \%$ of appropriate patients receive pharmacotherapy. ${ }^{4}$

In a review by Mauer et $\mathrm{al},{ }^{5}$ the authors conclude that pharmacotherapy aids in weight loss and prevents regain. However, analysis of a survey of 94 primary care physicians found $76 \%$ did not recommend pharmacotherapy for long-term weight loss, and 58\% had negative views towards pharmacotherapy. ${ }^{4}$ These data suggest that the prevailing philosophy is to avoid medications at all costs except in severe cases. ${ }^{4}$ Delaying pharmacotherapy until patients are severely obese may be too little, too late.

Recent guidelines suggest essential components of obesity care. ${ }^{1}$ Clinicians should ask the patient for permission to discuss obesity. Core tenets consist of individualized nutritional, physical, and psychological interventions along with surgery and medications. ${ }^{1}$ These guidelines also offer a new conceptualization of treatment that shifts the focus fRom BMI to improving overall health and wellbeing.

\section{Vania Modesto-Lowe, MD \\ Connecticut Valley Hospital \\ Addiction Services Division \\ Middletown, CT}

Allison Wick, BS

Middletown, CT

Michael Dang, BS, MD

Middletown, CT

\section{-REFERENCES}

1. Wharton S, Lau DCW, Vallis M, et al. Obesity in adults: a clinical practice guideline. CMAJ 2020; 192(31):E875-E891. https://www. cmaj.ca/content/192/31/E875. Accessed November 19, 2021.

2. Greaney ML, Cohen SA, Xu F, Ward-Ritacco CL, Riebe D. Healthcare provider counselling for weight management behaviours among adults with overweight or obesity: a cross-sectional analysis of National Health and Nutrition Examination Survey, 2011-2018. BMJ Open 2020; 10(11):e039295. doi:10.1136/bmjopen-2020-039295

3. Iwamoto S, Saxon D, Tsai A, et al. Effects of education and experience on primary care providers' perspectives of obesity treatments during a pragmatic trial. Obesity (Silver Spring) 2018; 26(10): 1532-1538. doi:10.1002/oby.22223

4. Granara B, Laurent J. Provider attitudes and practice patterns of obesity management with pharmacotherapy. J Am Assoc Nurse Pract 2017; 29(9):543-550. doi:10.1002/2327-6924.12481

5. Mauer Y, Parker M, Kashyap SR. Antiobesity drug therapy: an individualized and comprehensive approach. Clev Clin J Med 2021; 88(8):440-448. doi:10.3949/ccjm.88a.20080

doi:10.3949/ccjm.88c.12003 